\title{
Confirmation of the Cholinergic Specificity of the Chol-1 Gangliosides in Mammalian Brain Using Affinity-Purified Antisera and Lesions Affecting the Cholinergic Input to the Hippocampus
}

\author{
E. A. Derrington, ${ }^{*}$ D. Masco, and V. P. Whittaker \\ Arbeitsgruppe Neurochemie and ${ }^{*}$ Abteilung Neurobiologie, Max-Planck-Institut für \\ Biophysikalische Chemie, Göttingen, F.R.G.
}

\begin{abstract}
An antiserum raised to Torpedo electromotor synaptosomal membranes (anti-TSM antiserum) induces a cholinergic-specific immune lysis of mammalian brain synaptosomes and recognizes a group of minor gangliosides in mammalian brain. These minor gangliosides appeared, therefore, to be specific to the cholinergic neuron and were designated Chol-1. To confirm the cholinergic specificity of the Chol-1 gangliosidic antigens, we have shown that not only does a mammalian ganglioside fraction that is enriched with respect to the Chol-1 gangliosides inhibit the cholinergicspecific immune lysis induced by the anti-TSM antiserum. but also it can be used to affinity-purify a subpopulation of immunoglobulins from the anti-TSM antiserum that also induce a cholinergic-specific lysis. Furthermore, we have demonstrated that fimbrial lesions, which cause a massive degeneration of cholinergic terminals in the ipsilateral hippocam-
\end{abstract}

pus, lead to a loss of the Chol-1 gangliosides concomitant with that shown by choline acetyltransferase activity and that lesions to the entorhinal cortex, which cause a loss of mainly glutamergic synapses in the ipsilateral dentate gyrus leading to cholinergic sprouting from adjacent hippocampal areas and an increase in cholinergic markers in the dentate gyrus, produce concomitant increases in choline acetyltransferase activity and Chol- 1 content. These results provide strong evidence in favour of the cholinergic specificity of the Chol-1 gangliosides. Key Words: Chol-1 gangliosides-Cholinergicspecific antigens-Complement lysis-Ganglioside-Hippocampus. Derrington E. A. et al. Confirmation of the cholinergic specificity of the Chol-1 gangliosides in mammalian brain using affinity-purified antisera and lesions affecting the cholinergic input to the hippocampus. J. Neurochem. 53, 1686-1692 (1989).
In previous work in our laboratory, an antiserum [designated anti-TSM (Torpedo electromotor synaptosomal membranes)] has been raised against synaptosomal membranes prepared from the purely cholinergic electromotor innervation of the electric organ of Torpedo marmorata. This antiserum induced the specific complement-mediated lysis of the cholinergic subpopulation of mammalian brain synaptosomes, thereby indicating the presence of a cholinergic-specific cell surface antigen on mammalian nerve terminals that has been conserved from Torpedo to mammals (Jones et al., 1981; Richardson et al., 1982). Gangliosides extracted from Torpedo electric organ inhibited this complement-mediated lysis, and affinity purifica- tion of the antiserum on immobilized Torpedo electric organ gangliosides led to an increase in the capacity of the affinity-purified antiserum to induce the complement-mediated lysis of mammalian brain cholinergic synaptosomes (Ferretti and Borroni, 1986). It was accordingly inferred that the epitope recognized by Torpedo-affinity-purified anti-TSM antiserum in mammalian brain was also located on gangliosides. This putative cholinergic-specific gangliosidic antigen was named Chol-1. Further support for this hypothesis was derived from the demonstration by Ferretti and Borroni (1986) that the antiserum detected two immunoreactive bands in mammalian gangliosides (here designated Chol-1- $\alpha$ and $-\beta$ ) when the latter were separated by

Received October 7, 1988; revised manuscript received March 20 . 1989; accepted April 20, 1989

Address correspondence and reprint requests to Secretariat, $A G$ Neurochemie, Max-Planck-Institut für Biophysikalische Chemie, Postfach 2841, D-3400 Göttingen. F.R.G.

Abbreviations used: ChAT, choline acetyltransferase; ELISA, enzyme-linked immunosorbent assay; MAP, mammalian affinity-purified; PBS, phosphate-buffered saline; PSG, polysialoganglioside; TSM, Torpedo electromotor synaptosomal membranes. 
TLC and that a polysialoganglioside (PSG) fraction $\left(\mathrm{F}_{\mathrm{p}}\right)$ extracted from guinea-pig brain that was enriched in the immunoreactive bands was effective in inhibiting the complement-mediated lysis induced by Torpedoaffinity-purified anti-TSM antiserum.

We have sought to confirm the cholinergic specificity of the mammalian Chol-1 gangliosides in two ways: (a) by the application of appropriate immunochemical tests based on a mammalian PSG fraction enriched in the Chol-1 gangliosides and (b) by showing that degeneration of cholinergic synapses and cholinergic synaptogenesis brought about in the mammalian nervous system by surgical intervention led to the fall and rise in levels of Chol-1 gangliosides expected if these are indeed valid mammalian central cholinergic markers, as strongly indicated by recent immunohistochemical studies (Obrocki and Borroni, 1988).

The immunochemical tests showed that (a) the PSG fraction reduced the effectiveness of whole anti-TSM antiserum in inducing the specific complement-mediated lysis of the cholinergic subpopulation of mammalian brain synaptosomes and (b) antibodies to mammalian Chol-1 effective in inducing such lysis could be affinity-purified from the anti-TSM antiserum by adsorption onto an immobilized PSG and subsequent desorption.

The degeneration and regeneration studies used the cholinergic input to the hippocampus: Section of the fimbria causes its degeneration (Atack et al., 1987), and interruption of the entorhinal input to the dentate gyrus by causing a loss of noncholinergic innervation provokes axonal sprouting and reactive synaptogenesis in the cholinergic input (Lynch et al., 1972).

The quantitative assay of the Chol-1 gangliosides required by these studies used a recently developed modified enzyme-linked immunosorbent assay (ELISA) on TLC plates (E. A. Derrington and E. Borroni, manuscript submitted) referred to here as quantitative immune TLC.

\section{MATERIALS AND METHODS}

\section{Gangliosides}

PSGs. A PSG fraction from ox brain, designated PSG-4, was purchased from Dr. Pallmann KG (Munich, F.R.G.); it was estimated to be enriched in Chol- 1 antigens $\sim 40$ times relative to whole brain gangliosides and served as a Chol-1 standard. Its composition was determined by separating it by TLC and densitometrically scanning the bands revealed by application of Ehrlich's reagent $[6.0 \mathrm{~g}$ of $p$-dimethylaminobenzaldehyde (Sigma, Munich) dissolved in $800 \mathrm{ml}$ of ethanol and $200 \mathrm{ml}$ of $10 \mathrm{M} \mathrm{HCl}$. It was found to consist of $74 \%$ GT1b, 25\% GQ, and 1\% GD3 plus GDla plus GD1b. No evidence for the presence of nongangliosidic lipids in the mixture could be found when large amounts were separated and then stained with molybdenum blue reagent (Sigma) or by charring in the presence of sulphuric acid at $12^{\circ} \mathrm{C}$. No trace of protein contaminants could be found after separating $10 \mu \mathrm{g}$ in 10 or $15 \%$ polyacrylamide gels and staining with the highly sensitive silver reagent, nor were the Chol- 1 antigens sensitive to digestion by type $\mathrm{V}$ protease, type VI protease, or trypsin (E. A. Derrington and E. Borroni, manuscript submitted).

The bovine standard ganglioside mixture was a generous gift from Dr. A. Giuliani (Department of Medical Chemistry and Biochemistry, University of Milan, Milan, Italy). Its composition was determined to be $21 \%$ GM3, $33 \%$ GMI, $22 \%$ GDla, $14 \%$ GD lb, $8 \%$ GT 1 b, and $2 \%$ GQ. Gangliosides from tissue homogenates were extracted according to the method of Svennerholm and Fredman (1980), and their sialic acid content was determined by the method of Svennerholm (1957).

Chol-1 was present in both PSG-4 and the standard ganglioside mixture in concentrations too small to be detected by Ehrlich staining but was nevertheless considerably more concentrated there than in mammalian brain. For this reason, either of the two preparations could be used as standards to test for the linearity of the immune staining of TLC plates as a function of the amount of ganglioside applied. The precise structure of Chol-1 has not yet been determined.

Antiserum. This was the antiserum (anti-TSM) described by Richardson et al. (1982) and raised in a sheep to Torpedo electromotor synaptosomal plasma membranes derived from the electric organ.

Quantitative immune TLC. The Chol-1 content was estimated after separation on TLC plates by a modified ELISA procedure as follows (cf. E. A. Derrington and E. Borroni, manuscript submitted). Gangliosides containing between 2 and $3 \mu \mathrm{g}$ of sialic acid were applied in $20 \mu \mathrm{l}$ of chloroform/ methanol $(2: 1 \mathrm{vol} / \mathrm{vol})$ to aluminum-backed Kieselgel 60 "high-performance" TLC plates (Merck, Darmstadt, F.R.G.); the sialic acid content of the solution being applied was estimated according to the technique of Svennerholm (1957) in two 10- $\mu$ l samples. In this way, the effect of evaporation from the relatively small starting volumes of ganglioside/solvent solutions could be allowed for.

Gangliosides were separated by a one-dimensional chromatography in chloroform/methanol/0.2\% $\mathrm{CaCl}_{2}$ (50:40:11 by volume). After drying, the antigenic gangliosides were detected using anti-TSM antiserum by a modification of the method of Brockhaus et al. (1981). In brief, following incubation for $3 \mathrm{~h}$ with untreated anti-TSM antiserum, the TLC plate was extensively washed with $0.15 \% \mathrm{NaCl}$ in $10 \mathrm{mM}$ sodium phosphate buffer, $\mathrm{pH} 7.4$ [phosphate-buffered saline (PBS)], and a peroxidase-labelled rabbit anti-sheep immunoglobulin (Cappel Laboratories Dinatech, Plochingen, F.R.G.) was used to detect antibodies bound to gangliosides. The reaction was developed by dipping the chromatogram in $0.01 \%$ 4-dichloro-l-naphthol and $0.9 \mathrm{~m} M \mathrm{H}_{2} \mathrm{O}_{2}$ in PBS.

The immune staining was quantified by scanning with a Shimadzu CS-930 dual wavelength TLC scanner with a Shimadzu D2-2 data recorder. The linearity of the immune staining on each TLC was established (Fig. 1) by comparison with various amounts of a standard ganglioside mixture or PSG-4.

Because small changes in the composition of the solvent system due to evaporation cause changes in $R_{\mathrm{f}}$, Ehrlich stain was normally applied to the TLC plates after they had been subjected to immune staining to reveal the positions of the major gangliosides. This, however, lowered the sensitivity of the staining, particularly with the PSGs; thus, to obtain the quantitative distribution of the major gangliosides, other TLC plates were stained directly with Ehrlich reagent before scanning. 

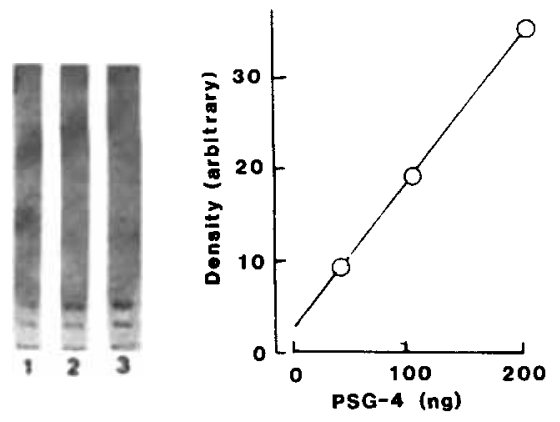

FIG. 1. Left: Lanes $1-3$ show 40,100 , and $200 \mathrm{ng}$, respectively, of PSG-4 separated by TLC and immunostained with the anti-TSM antiserum. Right: The thin-layer chromatogram was densitometrically scanned, and the area under the peaks was determined by the automatic peak detection function of the DR-2 data recorder to quantify the intensity of the immune staining. The graph shows that the intensity of the immune staining is a linear function of the quantity of PSG-4 applied to the plate.

Neuraminidase digestions. Mild neuraminidase digestion was performed using neuraminidase (type V, from Clostridium perfringens; Sigma) in a concentration of $1.5 \mathrm{U} / 50 \mu \mathrm{g}$ of ganglioside-bound sialic acid, dissolved in $50 \mathrm{mM}$ acetate buffer ( $\mathrm{pH} \mathrm{5}$ ), at $37^{\circ} \mathrm{C}$ for $18 \mathrm{~h}$. Stronger neuraminidase digestion was performed using the same enzyme but in 1 $\mathrm{m} M$ acetate buffer (pH 5 ) and $0.1 \%$ sodium taurodeoxycholate.

Affinity purification of antiserum. PSG-4 (10 mg) was covalently bound to glass beads of controlled pore size conjugated to a long-chain alkylamine (CPG/long-chain alkylamine; Pierce Chemical Co., Rockford, IL, U.S.A.) by the method of Young et al. (1978), except that owing to the low solubility of PSGs in pure dioxane, the reaction was carried out in dioxane/water (1:1 vol/vol). 1-Ethyl-3-(3-dimethylaminopropyl)carbodiimide in $N$-hydroxysuccinimide was used to form the activated esters of the gangliosides present. The immobilized gangliosides were loaded into a glass column closed at the end by a sintered glass disc. The column was washed extensively with PBS, and then whole anti-TSM antiserum $(50 \mu \mathrm{l}$ diluted in $1 \mathrm{ml}$ of PBS) was passed five times through the column. After further washing with PBS, bound antibodies were eluted with $1 \mathrm{ml}$ of $3 \mathrm{M} \mathrm{KSCN}$ followed by $2 \mathrm{ml}$ of water. The eluate was dialysed against $1 \%$ bovine serum albumin in PBS at $4^{\circ} \mathrm{C}$ for $12-15 \mathrm{~h}$ and stored at $-20^{\circ} \mathrm{C}$. The affinity-purified antiserum thus obtained is designated mammalian affinity-purified (MAP) antiserum.

Complement-mediated lysis. The ability of untreated and affinity-purified anti-TSM antisera to lyse cholinergic synaptosomes and that of fraction PSG-4 to inhibit the lysis induced by the untreated antiserum were tested according to the procedure of Ferretti and Borroni (1986).

\section{Experimentally induced perturbation of central cholinergic synapses}

Animals. Adult male albino rats of the Wistar strain, 3-6 months of age, were obtained from the breeding colony of the Max-Planck-Institut.

Lesions. Animals were anaesthetized by intraperitoneal injection of procaine $\left(6 \mathrm{mg} \mathrm{kg}^{-1}\right)$. Complete mechanical section of the fimbria and overlying cortex was performed ac- cording to the technique of Dravid and Van Deuson (1983). Electrolytic ablation of the entorhinal cortex was performed according to the method of Lynch et al. (1972).

Tissue preparation. At the appropriate time following lesioning, animals were killed by decapitation, and the ipsiand contralateral hippocampi were rapidly removed. In the case of fimbrial lesioning, whole hippocampi were homogenized in ice-cold PBS, and samples were taken for the determination of protein content and choline acetyltransferase (ChAT) activity. The remainder was stored at $-20^{\circ} \mathrm{C}$ for $12-$ $15 \mathrm{~h}$ before extraction of gangliosides. In the case of entorhinal lesioning, the hippocampi were cut into 14-16 500- $\mu$ m-thick slices using a Mcllwain tissue chopper; the most dorsal and ventral portions of the hippocampus were discarded. The hippocampal slices were then subdissected in ice-cold PBS with the aid of a Zeiss dissecting microscope into the dentate gyrus and the remaining tissue. Because of the very small amounts of tissue available, it was impossible to estimate Chol- 1 content and ChAT activity in the same animals; therefore, a sample was taken for the determination of protein content, and the remainder was either assayed for ChAT activity or stored for the extraction of gangliosides.

Protein and enzyme assays. Protein content was determined according to the procedure of Lowry et al. (1951), and ChAT activity was assayed according to the technique of Fonnum (1975). The immunoglobulin contents of MAP and whole anti-TSM antiserum were assayed by a solid-phase immunoassay as described by Ferretti and Borroni (1986).

\section{RESULTS}

\section{Immunochemical studies}

Characterization of a mammalian ganglioside fraction enriched in Chol-1. Figure 2 shows the separation on TLC of the ox brain PSG fraction PSG-4, before and after neuraminidase treatment. Two components,

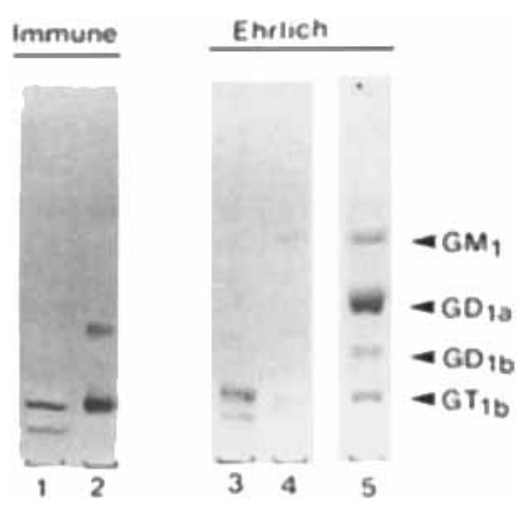

FIG. 2. Separation by TLC of a PSG fraction PSG-4 before (lanes 1 and 3) and after (lanes 2 and 4) mild neuraminidase treatment. The plates were immune stained with untreated anti-TSM antiserum, photographed (lanes 1 and 2), then sprayed with Ehrlich's reagent, and rephotographed (lanes 3 and 4); thus, lanes 1 and 3 and lanes 2 and 4 are identical, sequentially stained lanes of the TLC plate. The change in migration of the immunoreactive bands following neuraminidase treatment indicates the presence of sialic acid in these glycolipids and hence their gangliosidic nature. For reference, lane 5 shows a standard ganglioside mixture, stained with Ehrlich's reagent without prior immune staining. 
defined as Chol- $1 \alpha$ and - $\beta$, with $R_{\mathrm{f}}$ values a little lower than the main Ehrlich-positive GT1b and GQ bands, are strongly immunoreactive to untreated anti-TSM antiserum (lane 1) but are present in too low a concentration to give Ehrlich-positive bands. That these are nevertheless sialoglycolipids is shown by the fact that on mild neuraminidase treatment of PSG-4, their immunoreactivity is not destroyed, but their mobility is increased (lane 2). Stronger neuraminidase treatment (at low buffer concentrations and in the presence of sodium taurodeoxycholate) causes a complete loss of immunoreactivity (data not shown). A quantitative comparison of the intensity of immunostaining with that of appropriate amounts of a whole rat brain ganglioside preparation indicated that PSG- 4 is $\sim 40$-fold enriched in the immunoreactive gangliosides.

Antibodies recognizing mammalian Chol-1 gangliosides are cholinergic specific. To test whether antibodies that recognize the immunoreactive gangliosides are responsible for the immune lysis of cholinergic synaptosomes, we have used two approaches: First, we have tested whether preincubation of the whole anti-TSM antiserum with fraction PSG-4 leads to an inhibition of the antiserum's ability to lyse cholinergic synaptosomes; and second, we have affinity-purified the antiserum on immobilized PSG-4 and tested whether the antibodies that bound to and were eluted from the column are capable of inducing cholinergic complement lysis.

Figure 3 shows the results obtained when whole antiTSM antiserum is preincubated with PSG-4 before its use for complement-mediated lysis. The results indicate that there is a substantial loss in the ability of the serum to lyse cholinergic synaptosomes. This is presumably caused by the binding of anti-Chol- 1 antibodies to the Chol-1 gangliosides, thus rendering them unavailable for attaching components of the complement system to cholinergic synaptosomes. The failure of this inhibition to extend to $100 \%$ may have more than one

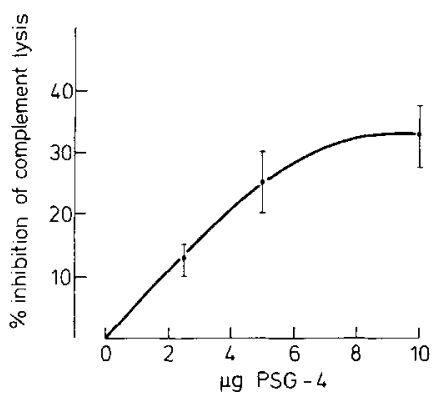

FIG. 3. Dose-dependent inhibition of cholinergic-specific complement-mediated lysis obtained by preincubation of anti-TSM antiserum with the mammalian PSG fraction PSG-4. The fact that a plateau is reached at a low level of inhibition may be due either to the existence of other cholinergic-specific antigens that are also recognized by the serum or to steric hindrance of epitopes caused by micellization at higher concentrations of PSG-4.
TABLE 1. Affinity purification of anti-TSM antiserum on a column of immobilized $P S G-4$

\begin{tabular}{lccc}
\hline $\begin{array}{l}\text { Anti-TSM } \\
\text { antiserum }\end{array}$ & Dilution & $\begin{array}{c}\text { IgG concentration } \\
\left(\mu \mathrm{g} \mathrm{ml}^{-1}\right)\end{array}$ & $\begin{array}{c}\text { Release of ChAT } \\
(\%)\end{array}$ \\
\hline Untreated & $1: 200$ & $226 \pm 17$ & $40 \pm 6$ \\
MAP & $1: 1$ & $26.1 \pm 1.3$ & $31 \pm 2$ \\
MAP & $1: 5$ & $5.2 \pm 0.26$ & $10 \pm 4$ \\
\hline
\end{tabular}

explanation. Thus, gangliosides form micelles in aqueous solution when they exceed a very low threshold concentration. Such micellization could hinder the binding of antibodies as a result of internalization of epitopes or surface steric effects. Alternatively, it may be that the Chol-1 gangliosides are not the only antigens recognized by the untreated anti-TSM antiserum on the surface of cholinergic synaptosomes.

To confirm that the epitope or epitopes on the gangliosides are indeed present on the rat brain cholinergic synaptosomes, we have tested the ability of the MAP anti-TSM antiserum to induce cholinergic-specific complement-mediated lysis, because such an antiserum should be uniquely enriched in antibodies that recognize Chol-1. Table 1 shows that MAP anti-TSM antiserum does indeed induce cholinergic-specific complement lysis. This result demonstrates that the ganglioside epitope or epitopes present in PSG-4 are indeed cholinergic specific and confirms and extends the earlier result of Ferretti and Borroni (1986) with Torpedoaffinity-purified anti-TSM antibodies.

\section{Effects of experimentally induced perturbation of central cholinergic synapses on Chol-1 content}

Experimental design. To supplement by quantitative studies the immunohistochemical evidence of Jones et al. (1981) and of Obrocki and Borroni (1988) that Chol1 gangliosides are located exclusively in cholinergic neurons of the mammalian CNS and especially their nerve terminals, we have experimentally perturbed the cholinergic input to the hippocampus in two ways: by causing them to degenerate and by inducing their proliferation.

Effect of degeneration. Unilateral lesions to the fimbria typically result in a massive loss 1-2 weeks postlesion of cholinergic markers (ChAT, acetylcholinesterase, and high-affinity choline uptake) ipsilateral to the lesion (Atack et al., 1987). We examined the effect of such lesions on the concentration of Chol-1 using the loss of the cholinergic-specific marker ChAT as an index for the extent of the degeneration of cholinergic nerve terminals; the contralateral hippocampus was used as a control. Figure 4 shows gangliosides extracted from hippocampi ipsi- and contralateral to fimbrial lesions after separation on TLC and immunostaining. No significant change was detected in the relative concentrations of the major brain gangliosides, which were found to be $23.5 \pm 2.0 \%$ GM1, 33.3 $\pm 1.7 \%$ GD1a, 


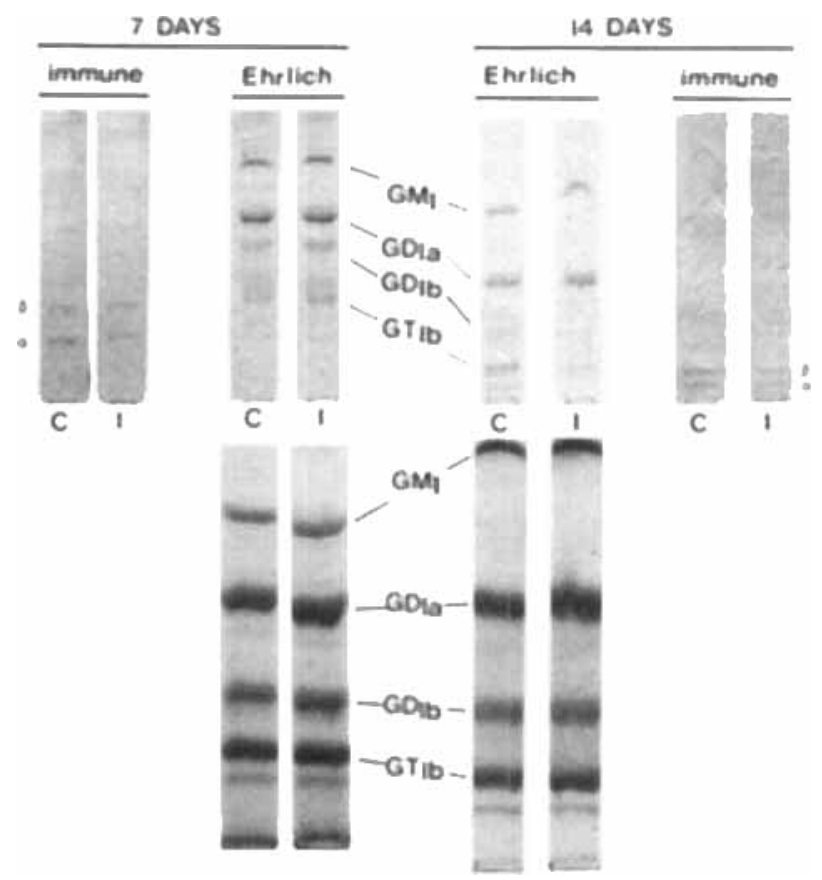

FIG. 4. Upper lanes: Gangliosides from hippocampi contralateral (C) and ipsilateral (l) to lesions of the fimbria 7 (left) and 14 (right) days postlesion have been separated on TLC and stained with anti-TSM antiserum ('immune') and subsequently with Ehrlich's reagent ("Ehrlich"). Two immunoreactive Choi-1 gangliosides are seen to have migrated just below the main GT1b and GQ ganglioside fractions and are labelled $\beta$ and $\alpha$, respectively. Lower lanes: These correspond to the ones immediately above them but are stained with Ehrlich's reagent without prior immune staining, the better to show the true relative intensities of the Ehrlich-positive bands. Slight changes in the composition of the solvent system due to evaporation explain the differences in $R_{t}$ between chromatographic assays.

$20.0 \pm 1.4 \%$ GD $1 \mathrm{~b}, 19.5 \pm 1.7 \%$ GT1b and $3.3 \pm 0.5 \%$ GQ. The concentration of Chol- 1 present was established by densitometric scanning and is shown in Fig. 5 together with ChAT activity, expressed as a percentage of the contralateral control. ChAT activity had fallen by $74 \pm 4 \%$ (mean \pm SEM; $n=6$ ) in the ipsilateral hippocampus relative to the contralateral control 7 days postlesion, whereas at this time the Chol- 1 content had fallen by $40 \pm 3 \%$ (mean \pm SEM; $n=6$ ). By 14 days postlesion, the Chol- 1 value fell to a level equivalent to that of ChAT, by $72 \pm 5 \%(n=6)$ and $71 \pm 5 \%(n=6)$, respectively. The slower time course for the loss of Chol- 1 is probably the result of a difference in the rate of metabolism of membrane glycolipids and soluble cytoplasmic proteins in degenerating terminals.

In contrast to lesions of the fimbria, which cause a degeneration of cholinergic synapses, lesions to the entorhinal cortex lead to the loss of mainly glutamergic synapses in the dentate gyrus. This causes the sprouting of cholinergic afferents in adjacent hippocampal areas; targets previously innervated by glutamergic synapses are reinnervated by cholinergic neurons mainly, although not exclusively, in the ipsilateral dentate gyrus (Lynch et al., 1972; Storm-Mathisen, 1974; Ulas et al., 1986). This phenomenon, known as reactive synaptogenesis, provides an opportunity to test the cholinergic specificity of the Chol-1 gangliosides, because the lesions cause a net loss of synapses but an increase in the number of cholinergic synapses.

The anti-Chol-1 immune reactivity was assayed in gangliosides extracted from the dentate gyrus and the rest of the hippocampus in unlesioned control animals and in animals 30 days postlesion ipsi- and contralateral to entorhinal lesions after separation on TLC (Fig. 6). ChAT activity was assayed similarly in other animals. The concentrations of Chol- 1 and ChAT in the various hippocampal areas in control and experimental animals are shown in Fig. 7. As expected, the formation of cholinergic synapses in the ipsilateral dentate gyrus caused a marked increase in the expression of ChAT in this area, which is parallelled by the increase in Chol1 content. The relative concentration of the major gangliosides in both the dentate gyrus and the rest of the hippocampus showed very little change as a result of the entorhinal cortex lesion except for a slight fall relative to unoperated controls in the concentration of GQ both ipsi- and contralateral to the lesion. This is further support for the cholinergic specificity of Chol1. Smaller changes occur in other hippocampal areas, particularly in the contralateral dentate gyrus; such changes in the contralateral hippocampus have been reported previously (Ulas et al., 1986). It is interesting to note, however, that the relative concentrations of Chol-1 and ChAT between areas in control and lesioned animals correlate quite closely with each other.

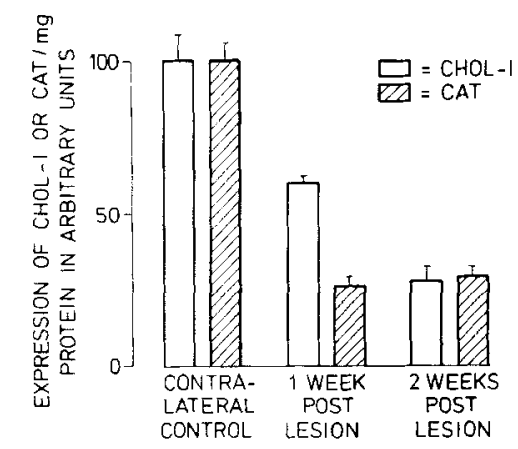

FIG. 5. Amounts of Chol-1 and ChAT (CAT) per milligram of protein present in the ipsilateral hippocampus expressed as percentages of the contralateral value. Although the Chol-1 immune reactivity falls more slowly than ChAT activity and appears to take twice as long to reach the same level, it is clear that the deafferentation of the major cholinergic input to the hippocampus causes a fall in Chol-1 content concomitant with that of ChAT activity. An almost identical profile is obtained if the results are expressed per milligram of ganglioside sialic acid. Data are mean \pm SD (bars) values of six experiments. 


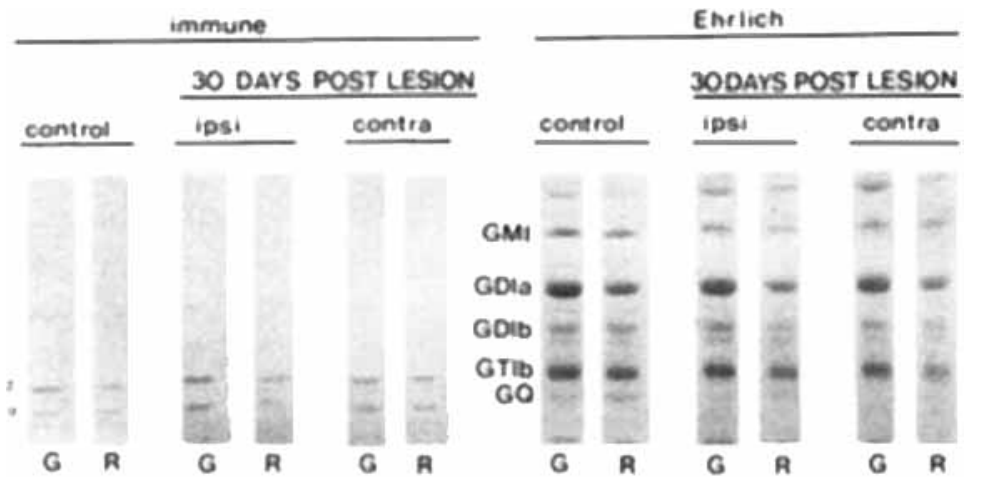

FIG. 6. TLC separation of gangliosides extracted from the dentate gyrus $(G)$ and the rest of the hippocampus $(R)$, ipsi- and contralateral to entorhinal lesions 30 days postlesion and from unlesioned control animals. The plates were stained with anti-TSM antiserum ("immune') followed by Ehrlich's reagent ("Ehrlich"). The Chol-1 gangliosides can be seen in the immune-stained plates migrating just below GT1b and GQ and are labelled $\beta$ and $\alpha$, respectively.

\section{DISCUSSION}

Previous work in this laboratory has shown that there are epitopes present on Torpedo electric organ gangliosides that are specific for the cholinergic subpopulation of mammalian brain synaptosomes. Some of these epitopes are also present in minor gangliosides in the mammalian brain that have been designated Chol-1. To establish that the mammalian Chol-1 gangliosides are indeed cholinergic specific, we have shown that (a) preincubation of the anti-TSM antiserum with the antigenic mammalian brain gangliosides inhibits its ability to lyse cholinergic synaptosomes, (b) antiTSM antiserum affinity-purified on immobilized mammalian brain gangliosides enriched with respect to the Chol-1 gangliosides lyses the cholinergic subpopulation of mammalian brain synaptosomes, (c) lesions that result in the degeneration of cholinergic synapses, as measured by the loss of ChAT, produce a concomitant loss of Chol-1, and (d) lesions that result in a net loss of synapses but a net increase in cholinergic

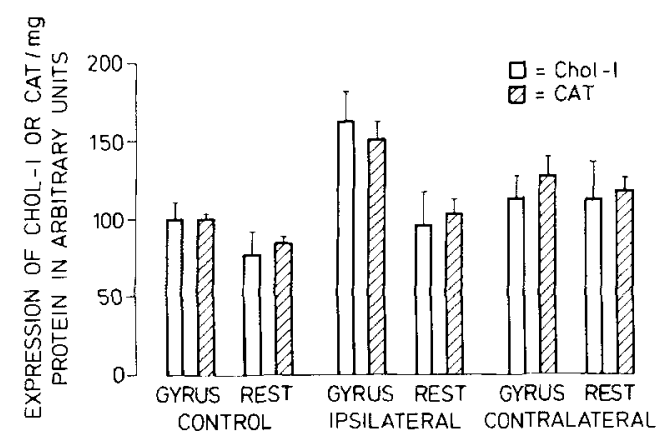

FIG. 7. Amounts of Chol-1 and ChAT (CAT) present per milligram of protein in the dentate gyrus and the rest of the hippocampus in control animals and ipsi- and contralateral to entorhinal cortical lesions 30 days postlesion. Results are expressed as percentages of the concentration in control gyri. As expected, there is a significant increase in ChAT activity caused by the formation of new cholinergic synapses in the ipsilateral dentate gyrus 30 days postlesion; this is paralleled by a rise in Chol-1 content. An almost identical profile is obtained with results expressed per miligram of ganglioside sialic acid (cf. Fig. 5). Data are mean \pm SD (bars) values of six experiments. synapses result in an increase in the Chol-1 concentration in the affected region, which parallels that of ChAT activity.

All of these results provide convincing new evidence that the mammalian Chol-1 gangliosides are indeed cholinergic specific.

Because Chol-1 has been defined as a cholinergicspecific ganglioside or gangliosides (Richardson et al., 1982), we believe that it is now important for clear evidence of specific immunoreactivity with mammalian Chol-1 gangliosides to be demonstrated before an antiserum is described as anti-Chol-1.

Although cell surface carbohydrates have been demonstrated to be specific markers for various neural cell types (Raff et al., 1978) and subclasses of cell types (Dodd and Jessell, 1985), the Chol-1 gangliosides are, so far, unique in that they are specific for neurons of one transmitter type. It is intriguing to speculate that other minor gangliosides may eventually be found that extend this kind of specificity to other transmitters.

Acknowledgment: We are most grateful to Dr. E. Borroni for much discussion and many useful suggestions. This work was supported by grants from the German Research Society (Wh 1/5-1) and from Fidia Pharmaforschung GmbH. To both these sponsors we express our sincere gratitude.

\section{REFERENCES}

Atack J. R., Perry E. K., Bonham J. R., Boakes R., and Candy J. M. (1987) Loss and recovery of acetylcholinesterase molecular forms in the fornix-lesioned rat hippocampus. Neurosci. Lett. 79, 174184.

Brockhaus M., Magnani J. L., Blaszczyck S. Z., Koprowski H., Karlsson K.-A., Larson G., and Ginsburg V. (1981) Monoclonal antibodies directed against the human $\mathrm{Le}^{\mathrm{b}}$ blood group antigen. J. Biol. Chem. 256, 13223-13225.

Dodd J. and Jessell T. (1985) Lactoseries carbohydrates specify subsets of dorsal root ganglion neurons projecting to the superficial dorsal horn of rat spinal cord. J. Neurosci. 5, 3278-3294.

Dravid A. R. and Van Deuson E. B. (1983) Recovery of choline acetyltransferase and acetylcholinesterase activities in the ipsilateral hippocampus following unilateral partial transection of the fimbria. Brain Res. 277, 169-174.

Ferretti P. and Borroni E. (1986) Putative cholinergic-specific gangliosides in guinea-pig forebrain. $J$. Neurochem. 46, 1888-1894.

Fonnum F. (1975) A rapid radiochemical method for the determination of choline acetyltransferase. $J$. Neurochem. 24, 407-409. 
Jones R. T., Walker J. H., Richardson P. J., Fox G. Q., and Whittaker V.P. (1981) Immunochemical localization of cholinergic nerve terminals. Cell Tissue Res. 218, 355-373.

Lowry O. H., Rosebrough N. J., Farr A. L., and Randall R. J. (1951) Protein measurement with the Folin phenol reagent. J. Biol. Chem. 193, 265-275.

Lynch G., Mathews D. A., Mosko W., Parks T., and Cotman C. (1972) Induced acetylcholinesterase-rich layer in rat dentate gyrus following entorhinal lesions. Brain Res. 42, 311-318.

Obrocki J, and Borroni E. (1988) Immunochemical evaluation of a cholinergic-specific ganglioside antigen (Chol-1) in the central nervous system of the rat. Exp. Brain Res. 72, 71-82.

Raff M. C., Fields K. L., Hakomori S. I., Mirsk R., and Pruss R. M. (1978) Cell-type specific markers for distinguishing and studying neurons and major classes of glial cells in culture. Brain Res. 174, 283-308.

Richardson P. J., Walker J. H., Jones R. T., and Whittaker V. P. (1982) Identification of a cholinergic-specific antigen Chol-1 as a ganglioside. $J$. Neurochem. 38, 1605-1614.
Storm-Mathisen J. (1974) Choline acetyltransferase and acetylcholinesterase in fascia dentata following lesion of the entorhinal afferents. Brain Res. 80, 181-197.

Svennerholm L. (1957) Quantitative estimation of sialic acids. II. A colorimetric resorcinol-hydrochloric acid method. Biochim. Biophys. Acta 24, 604-611.

Svennerholm L. and Fredman P. (1980) A procedure for the quantitative isolation of brain gangliosides. Biochim. Biophys. Acta 617, 97-109.

Ulas J., Gradkowska M., Jezierska M., Skup M., Skangiel-Kramska J., and Oderfeld-Nowak B. (1986) Bilateral changes in glutamate uptake, muscarinic receptor binding and acetylcholinesterase level in the rat hippocampus after unilateral entorhinal cortex lesions. Neurochem. Int. 9, 255-263.

Young W. W., Laine R. A., and Hakomori S. I. (1978) Covalent attachment of glycolipids to solid supports and macromolecules, in Methods in Enzymology, Vol. 50 (Ginsburg V., ed), pp. 137140. Academic Press, New York. 